RIVAS MARTÍNEZ, S. y P. CUBAS -1987- Ulex, in B. VALDÉS et al. (eds.) Flora Vascular de Andalucía Occidental 2: 165-169. Editorial Ketres. Barcelona.

ROMERO ZARCO, C. -1987- Medicago, Holcus, Agrostis, in Flora Vascular de Andalucia Occidental 2: 117-127, 3: 331-333, 336-341. Editorial Ketres. Barcelona.

RUÍZ DE CLAVIJO, E. - 1987- Trifolium, Osyris, in B. VALDÉS et al. (eds.) Flora Vascular de Andalucía Occidental 2: 97-116, 211-212. Editorial Ketres. Barcelona.

SILVESTRE, S. - 1987- Lythrum, in B. VALDÉS et al. (eds.) Flora Vascular de Andalucía Occidental 2: 193-198. Editorial Ketres. Barcelona.

TALAVERA, S. -1987- Moehringia, Matricaria, Glossopappus, Gymnostyles, Aetheorhiza, Staehelina, Hainardia, Brachypodium, Hordeum, Stipa, in B. VALDÉS et al. (eds.) Flora Vascular de Andalucia Occidental 1 : 231, 3: 50-51, 62-63, 69, 91-92, 175, 299, 369-372, 378-380, 381-384. Editorial Ketres. Barcelona. UBERA, J. L. -1987- Lamium, in B. VALDÉS et al. Flora Vascular de Andalucía Occidental 2: 409411. Editorial Ketres. Barcelona.
VALDÉS, B. -1987- Convolvulus, Linaria, Kickxia, Globularia, Asphodelus, Iris, in B. VALDÉS et al. (eds.) Flora Vascular de Andalucía Occidental 2: 363-369, 508-522, 525-528, 548-549, 3: 425-427, 477-482. Editorial Ketres. Barcelona.

VALDÉS, B., S. TALAVERA y E. FERNÁNDEZ GALIANO (eds.) -1987- Flora Vascular de Andalucía Occidental. 3 vols. Editorial Ketres. Barcelona.

VARELA, J. A., E. DOMÍNGUEZ y E. RUÍZ DE CLAVIJO -1981- Notas sobre la Flora de Córdoba. I. Algunas especies interesantes de los tramos medio e inferior del valle del Río Guadiato. Anales Jardín Botánico de Madrid, 38(1): 320-322.

Aceptado para su publicación en Abril de 1998

Dirección de los autores. Departamento de Biología Vegetal y Ecología. Facultad de Ciencias. Universidad de Córdoba. 14004 - Córdoba.

\title{
62. CISTUS LADANIFER L. SUBSP. AFRICANUS DANS., UN NUEVO TAXON PARA ANDALUCÍA OCCIDENTAL
}

Pablo GARCÍA MURILLO y Manuela PALACIOS

Cistus ladanifer subsp. africanus Dans., a new taxon in West Andalusia

Palabras clave. Cistaceae, Cistus, Andalucía Occidental.

Key Words. Cistaceae, Cistus, West Andalusia.

En el tratamiento de Cistus L. que realizaron Demoly y Montserrat para la Flora Ibérica (1993), reconocieron tres subespecies dentro de C. ladanifer L.: la subsp. ladanifer, abundante en el oeste de la Península, la subsp. africanus Dans., indicada sólo para Málaga y la subsp. sulcatus (Demoly) P. Monts., referida al Algarve portugués. 


\begin{tabular}{|l|c|c|}
\hline CARACTERES & $\begin{array}{c}\text { subsp. africanus } \\
\text { (población Tajo de las } \\
\text { Figuras. Cádiz) }\end{array}$ & $\begin{array}{c}\text { subsp. ladanifer } \\
\text { (según datos de } \\
\text { Cabezudo, 1987) }\end{array}$ \\
\hline $\begin{array}{l}\text { Longitud total de hojas (LT) } \\
\text { Longitud del limbo (L) }\end{array}$ & $30.7-65 \mathrm{~mm}$ & $40-110 \mathrm{~mm}$ \\
Anchura del limbo (A) & $24.6-52.7 \mathrm{~mm}$ & $40-110 \mathrm{~mm}$ \\
Longitud del pecíolo (P) & $7.2-12.3 \mathrm{~mm}$ & $10-20 \mathrm{~mm}$ \\
Razón L/A & $5-8.3 \mathrm{~mm}$ & - \\
Razón LT/P & $3.08-4.7$ & $4-5.5$ \\
Margen de la hoja & $4.84-9.27$ & - \\
Ládano & ligeramente revoluto & ligeramente revoluto \\
Nervios aparentes del envés & ligeramente ondulado & entero \\
Inflorescencias & presente & presente \\
número de brácteas & 1 & 1 \\
pubescencia sépalos & axilares & terminales \\
tamaño pétalos & $8-10$ & 4 \\
color de los pétalos & escamosa estrellada & escamosa estrellada \\
$\mathbf{n}^{\circ}$ lóculos del ovario & blancos sin mácula & $40-55$ mm \\
pubescencia capsula & $6-7$ & blancos con mácula o sin ella \\
\hline
\end{tabular}

Tabla 1. Caracteres morfológicos. Morphological characters.

Recientemente se localizó una población de Cistus ladanifer en el sur la provincia de Cádiz (Benalup de Sidonia, alrededores de la Cueva del Tajo de las Figuras, 30STF5422) cuyos individuos llamaron nuestra atención al presentar caracteres que se apartaban de los típicos de la subsp. ladanifer, la única citada para Andalucía Occidental por Demoly y Montserrat (1993). Las plantas mostraban las hojas claramente pecioladas, sus flores se situaban en las axilas de las hojas, soportadas por numerosas brácteas y las cápsulas presentaban 6-7 lóculos; a diferencia de los atributos característicos de la subsp. ladanifer: hojas sentadas, flores terminales, con pocas brácteas y cápsulas con 10 lóculos. La población observada era homogénea y se extendía por todo el sotobosque del alcornocal aclarado que rodea los riscos del Tajo de las Figuras. El sustrato sobre el que se asentaba la población estaba formado por areniscas y acompañando a esta jara se observaron:
Calicotome villosa (Poiret) Link, Genista triacanthos Brot., Teline linifolia (L:) Webb \& Berth., Rubus ulmifolius Shott, Myrtus communis L., Rhamnus lycioides L., Adenocarpus telonensis (Loisel.) DC., Phyllirea angustifolia L., Osyris quadripartita Salzm., Erica australis L., como las especies más conspicuas del matorral

Tras el examen detenido de sus caracteres, resumidos en la tabla 1 , las plantas se identificaron con Cistus ladanifer subsp. africanus Dans., lo que constituye una novedad para la Flora de Andalucía Occidental.

Según Danserau (1951), autor de la descripción de este taxon, se trata de la subespecie de Cistus ladanifer que se extiende ampliamente por el NW de Africa: Argelia y Marruecos, donde fue recolectado hace algunos años por uno de los autores de esta nota (ver relación de material estudiado y referencia en artículo de Arista y Ortiz, 1994). Y, si bién, el criterio de Danserau (1951) ha sido considerado en algunas obras capitales (Greuter et al., 
1984), ha sido rechazado por Fennane \& Matez (1992), al no hallar, estos autores, una separación neta con la subespecie típica y detectar algunas poblaciones de africanus en el sur de España. Nosotros, tras haber estudiado minuciosamente los caracteres de la población gaditana y, posteriormente, diverso material de herbario procedente del sur de España y norte de África, pensamos que la separación e identidad de esta subespecie no ofrece lugar a dudas. Asimismo, teniendo en cuenta las características de las localidades indicadas en la relación de material estudiado, señalamos que C. ladanifer subsp. africanus, en España, no es un taxon de serpentinas, como apuntan Demoly y Montserrat (1993), sino que ocupa también sustratos procedentes de rocas areniscas.

Material estudiado (se incluye aquí el material indicado amablemente por el Prof. P. Montserrat, in littera, y que se destaca con un asterisco *).

ESPAÑA. Cádiz. Alcalá de los Gazules, 13-IX1929, H. del Villar (MA 159625); Algeciras, Sierra del Rompecoche, 5-V-1907, R. Chodat (* G); Benalup de Sidonia, alrededores de la cueva del Tajo de las Figuras, 8-XII-1996, P. García Murillo y M. Palacios (SEV150286); idem, 7-III-1998, P. García Murillo y M. Palacios (SEVF); Castellar de la Frontera, 80 m, 21-IV-1972, P. Geissler (* G); Medina Sidonia, cerro Almazán, areniscas (* MGC30205); Montes de Jerez, Sierra de la Gallina, 3-III-1973, B. Casaseca (* SALA). Málaga. Benarraba, in collibus arenosis, 17-V-1932, C. Vicioso (MA79728); Benahavis, río Guadalmina, UF1546, $240 \mathrm{~m}$, serpentinas (* MGC44118); Casares, Sierra Bermeja, garganta Andías, UF0037 (* MGC44120); Estepona, Puerto de Peñas Blancas, sin fecha, S. Rivas Martínez? (MA322684); ídem, Sierra Bermeja, serpentinas, 400 m (* MGC31487); ídem, esquistos (* MGC31479); idem, los Reales (* MGC44119); Istán, Albornoque, WF2754 (* MGC31097); ídem, Sierra Real, P. Armas, $1250 \mathrm{~m}$, serpentinas (*MGC43283); Sierrade Gen (*MGC10182); Sierra de Nerja, VF27 (* MGC10773); Tolox, Sierra Parda, 780 m, serpentinas, VF2959 (* MGC37704).
MARRUECOS. Atlas Rifain, Bab du Cocteau, 1700 m, 13-VI-1935, Sennen y Mauricio (MA162637); Ben Ahmed Beni-Bouzesa, 535 m (* MGC42004); Beni-Hadifa, sol schisteux, 7-V, Sennen y Mauricio, Pl. D'Espagne 1931, n7785 (MA79685); Foret de M'Sil, IV-1922, Ch. d'Alleizette (MA79697); Ketama, 1650 m, pizarras El Bintz, 280 m, areniscas (* MGC28184); Rif oriental, Adrar Kerker, 22-III1956, J. Ruiz de la Torre; Supra Targuist, habitat in Quercetis, 1100 m. 22-V-1927, P. Font Quer, Iter Maroccanum 1927, nº 398 (MA79683); Taza, Djebel Arekdi, 1440 m, pizarras, 26-V1981, S. Castroviejo et al. (MA227520); Tanger, entre Tánger y Cabo Espartel, 35²4' N y 5 53'W, 160 m, 29-X-1993, $P$. García Murillo et al. (SEV135460); Uad el Jolot, areniscas El Bintz, $280 \mathrm{~m}$, areniscas (*MGC28135); Yutzam, sin fecha, M. Martínez (MA79701).

AGRADECIMIENTOS. Los autores desean agradecer al Prof. P. Montserrat la información facilitada sobre material del sur de España y norte de Africa.

\section{BIBLIOGRAFÍA}

ARISTA, M. y P. ORTIZ-1994- Números cromosomáticos de Plantas Occidentales. Números 681-687. Anales Jard. Bot. Madrid 51: 279.

CABEZUDO, B. -1987- Cistus L., in Valdés et al. (Eds.) Flora Vascular de Andalucía Occidental. I. pp. 335-340.

DANSERAU, P. -1951- Notes sur les Cistes. II. Revision de la section Ladanium. Mem. Soc. Bot. Fr. 32: 3-10.

DEMOLY, J. y P. MONTSERRAT- 1993- Cistus L., in Castroviejo et al. (Eds.) Flora Ibérica. III. Madrid. pp. 319-337.

FENNANE, M. \& J. MATHEZ (Eds.)-1992Noveaux materiaux pour la flore du Maroc. Fasc. 4. Nat. Monsp. Ser. Bot. 56: 151-176.

GREUTER, W., H.M. BURDET \& G. LONG (Eds.)1984-Med Checklist.I. Geneve. pp. 316.

Aceptado para su publicación en Marzo de 1998

Dirección de los autores. Dpto. Biología Vegetal y Ecología. Facultad de Farmacia. Universidad de Sevilla. Apdo. 874. 41012, SEVILLA. 